\title{
Cerebral Abscesses Resulting from H1N1 Influenza with Staphylococcal Co-Infection
}

\author{
Derek J. Roberts, John J.P. Kelly, Rajiv Midha, Aleksa Cenic
}

Can. J. Neurol. Sci. 2011; 38: 147-150

In 2009, a novel swine influenza A (H1N1) virus, which was capable of infecting humans and producing respiratory disease, was discovered in Mexico. ${ }^{1}$ Since the emergence of this virus, there have been multiple large, localized epidemics of infection in both Canada and the United States. ${ }^{1}$ Many of these epidemics were linked with increased rates of both critical illness and death, especially among patients that were young and otherwise healthy. ${ }^{1}$

During prior influenza pandemics, bacterial co-infection, particularly secondary bacterial infection of the lung, was associated with elevated morbidity and mortality. ${ }^{2,3}$ This association also now exists for those infected with the novel influenza A (HIN1) virus, as bacterial co-infection among these patients is linked with higher rates of both critical illness and death. ${ }^{3,4}$ Indeed, secondary bacterial pneumonia was reported in $24.4 \%$ of patients with H1N1 influenza admitted to Canadian intensive care units (ICUs). ${ }^{4}$

Pneumonia following H1N1 influenza is commonly due to Staphylococcus aureus with the resultant bacterial sepsis being a leading cause of death. ${ }^{4,5} \mathrm{~S}$. aureus is an invasive bacterium associated with significant pulmonary parenchymal destruction and lung abscess formation. Lung abscesses can lead to bacteremia, which in some patients, may result in seeding of well-perfused solid organs, including brain. Despite this, cerebral infection and/or abscess formation has not yet been described in association with influenza infection.

In this report, we present a unique case of a methicillinsensitive $S$. aureus (MSSA) co-infection that resulted in bacteremia, cerebral abscesses, and coma in a critically ill patient with the novel influenza A (H1N1) virus. The clinical course, imaging, and outcome of this patient are presented in detail. We also hypothesize on the pathophysiologic link between cerebral abscesses and bacterial co-infection in a patient with severe viral infection, as well as provide a brief overview on the current management strategies for cerebral abscesses.

\section{The Case}

A previously healthy 19-year-old male presented with an altered level of consciousness preceded by several hours of severe headache and confusion. Ten-days prior, he had begun experiencing mild headaches with malaise, fatigue, rhinorrhea, myalgias, and fever. On examination, the patient was febrile with a temperature of $40.3^{\circ} \mathrm{C}\left(104.5^{\circ} \mathrm{F}\right)$; his remaining vital signs were within normal limits. The Glasgow Coma Scale (GCS) score was 8 (E2, V1, M5). The patient's pupils were 3-mm and reacted to light. All four extremities moved symmetrically and localized to painful stimuli while plantar responses were up- going. Neither nuchal rigidity nor peripheral stigmata of infective endocarditis were evident. Brudzinski's and Kernig's sign were absent. Auscultation of the heart and lung fields revealed normal heart sounds without murmurs, and decreased air entry with bronchial breathing and inspiratory crackles over the right lower lobe. The patient was admitted to the ICU and underwent endotracheal intubation followed by physiologic stabilization.

Numerous investigations were subsequently performed. A complete blood cell count revealed leukocytosis (white blood cell count of $18.9 \times 10^{9} / \mathrm{L}$ ). Computed tomography (CT) of the chest, abdomen, and pelvis demonstrated a left suprahilar lung abscess with adjacent areas of pulmonary consolidation, mediastinal lymphadenopathy, bilateral renal abscesses, three liver abscesses, and splenomegaly (Figure 1). Both transthoracic and transesophageal echocardiography were unremarkable. Flexible bronchoscopy revealed a left-sided lung abscess. Although nasopharyneal swab cultures were negative, bronchoalveolar lavage fluid samples were positive for Influenza A; this was subsequently confirmed by polymerase chain reaction to be the novel pandemic H1N1 strain. Both bronchoalveolar lavage fluid and blood cultures also grew MSSA. Human immunodeficiency virus type 1 and 2 and hepatitis $\mathrm{B}$ and $\mathrm{C}$ assays were all negative.

To evaluate the patient's depressed level of consciousness, a CT scan of the head was performed. This revealed several bilateral, intracerebral, hemorrhagic lesions with surrounding peri-lesional edema in the frontal, parietal, and occipital lobes (Figure 2). Subsequent plain and gadolinium-enhanced magnetic resonance imaging (MRI) of the brain demonstrated multiple well-circumscribed, ring-enhancing lesions felt to be in keeping with cerebral abscesses (Figure 3).

The patient was treated with oseltamivir, $150 \mathrm{mg}$ twice daily, together with cloxacillin, 2 grams intravenously, every fourhours. After several days, his level of consciousness began to improve. However, the cerebral abscesses continued to enlarge and develop progressive peri-lesional edema on serial head CT scans (Figure 4). A CT of the head performed nine-days after

From the Department of Surgery (DJR) and the Division of Neurosurgery (JJPK, RM, AC). University of Calgary and the Foothills Medical Centre, Calgary, Alberta, Canada.

Received July 13, 2010. Final Revisions Submitted August 25, 2010 Correspondence to: Aleksa Cenic, Division of Neurosurgery, Department of Clinical Neurosciences, Foothills Medical Centre, 1403-29th Street NW, Calgary. Alberta T2N 2T9, Canada. 

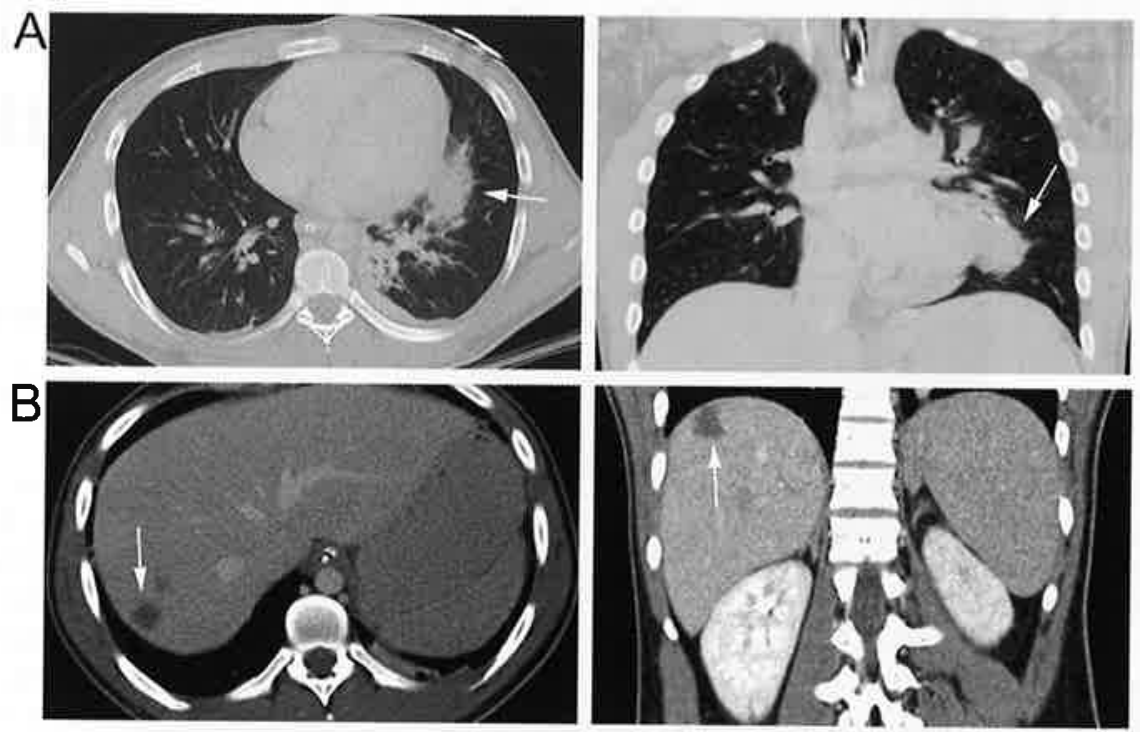

Figure 1: Axial and coronal computed tomography images of the chest $(A)$ and abdomen $(B)$ showing an abscess in the suprahilar region of the left upper lobe of the lung and the right lobe of the liver (arrows).

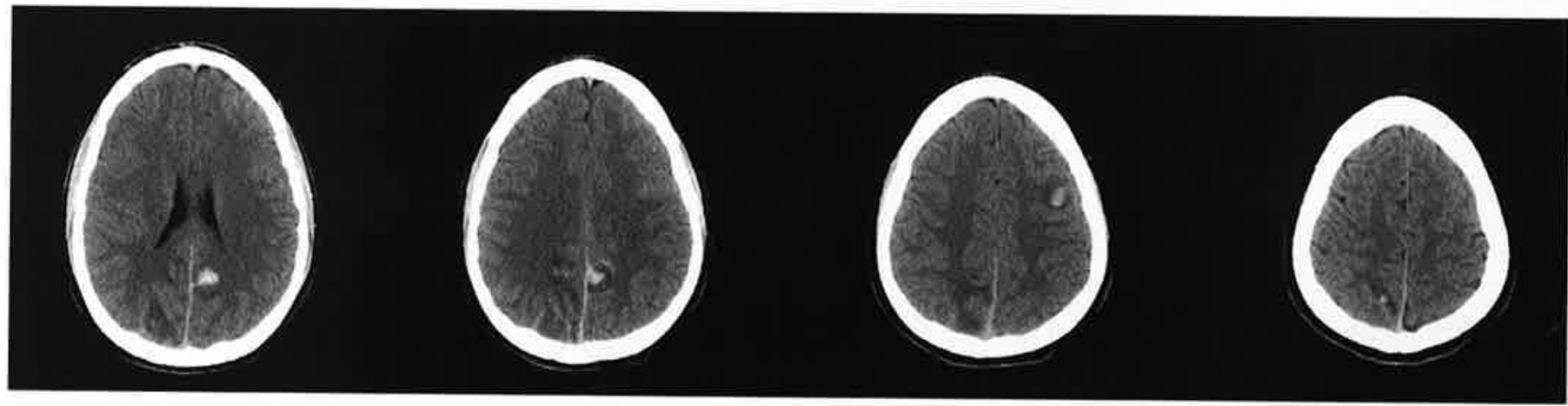

Figure 2: Presenting axial contrast-enhanced computed tomography images of the brain demonstrating multiple hemorrhagic intracerebral lesions with minimal surrounding peri-lesional edema.

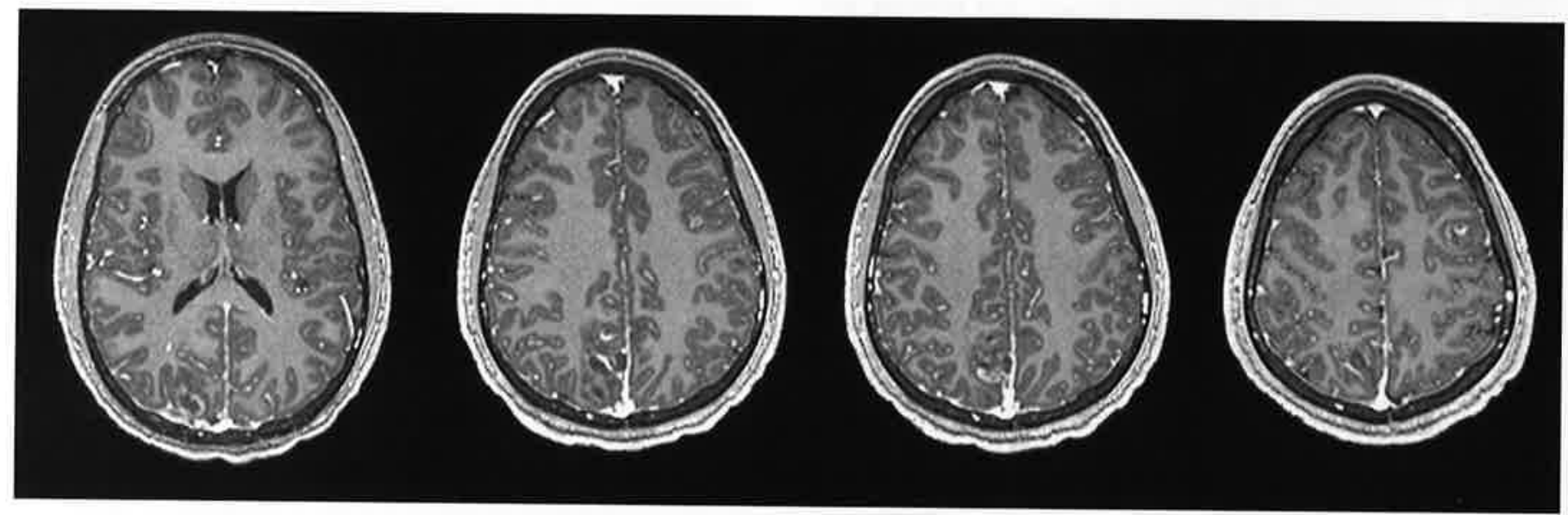

Figure 3: Presenting axial T1-weighted magnetic resonance images of the brain demonstrating several ring-enhancing intracerebral lesions consistent with cerebral abscesses. 


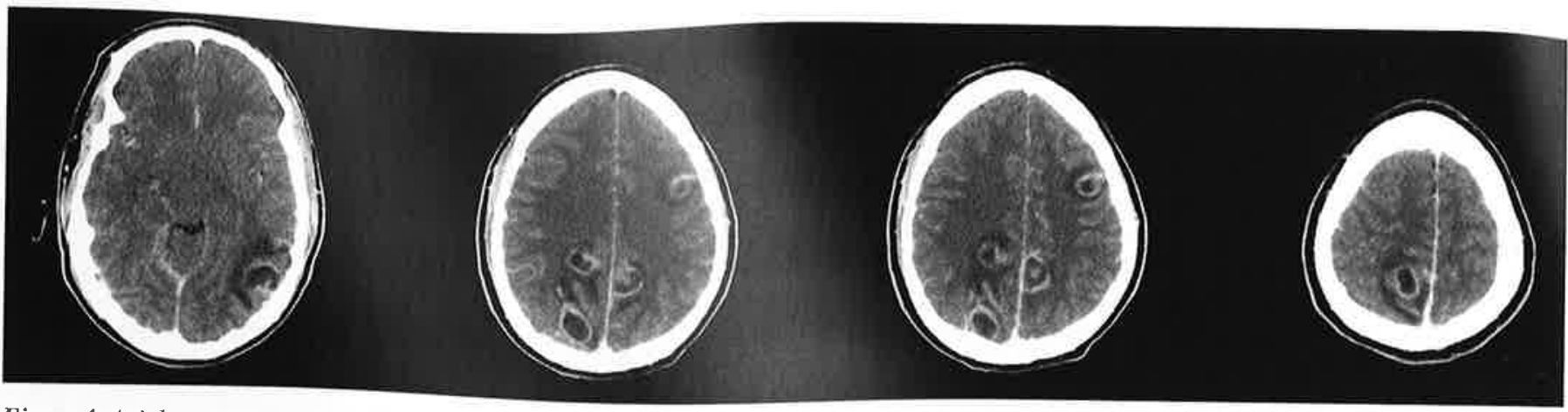

Figure 4: Axial contrast-enhanced computed tomography images of the brain nine-days after presentation revealing progressive growth and maturation of the previously identified cerebral abscesses despite antibiofic and antiviral therapy.

presentation showed radiological progression with more than ten cerebral abscesses, including a dominant parietooccipital abscess $(2.4-\mathrm{cm}$ by $2.3-\mathrm{cm})$. The neurological status of the patient, however, continued to improve daily.

The patient was extubated 11-days after presentation. He was initially disoriented to place and time and exhibited repetitive mannerisms and speech. Following inpatient speech, occupational, and physical therapy, the patient was discharged home on six-weeks of cloxacillin (two-weeks of outpatient IV followed by four-weeks of oral). At follow-up, seven-weeks after discharge, the patient's headaches were improving, his neurological deficits were undetectable, and the abscesses had nearly resolved on MRI (Figure 5). At the 12-week follow-up appointment, he had completed antibiotic therapy and had no headaches with a normal neurological examination. The patient returned to work and provided written informed consent for publication of this report.

\section{DiscUSSION}

This report describes the case of an otherwise-healthy 19 year-old critically ill patient that developed bacteremia and multiple cerebral and other solid organ abscesses following pulmonary co-infection with MSSA and the novel influenza A (H1N1) virus. The patient was managed medically with oseltamivir and target antibiotics and at seven-weeks posthospital discharge had no identifiable neurological deficits and was seizure-free. To our knowledge this is the first report of such an association and provides a novel example of the morbidity associated with bacterial co-infection in patients with novel influenza $\mathrm{A}(\mathrm{H} 1 \mathrm{~N} 1)$ infection.

Although unproven, the pathophysiology or etiology of brain abscess formation following bacterial co-infection is likely due, at least in part, to an influenza virus-associated alteration in blood-brain barrier function. Brain abscess formation requires the presence of pre-existing neural parenchymal lesions; they do not form following transient bacteremia in the absence of altered blood-brain barrier function or blood-brain barrier disruption..$^{6-8}$ For example, formation of cerebral abscesses in patients with congenital heart disease (a classic, but now uncommon, etiology) is secondary to development of cerebral microinfarctions due to increased blood viscosity from chronic hypoxemia as a result of left-to-right shunting. ${ }^{8}$ These

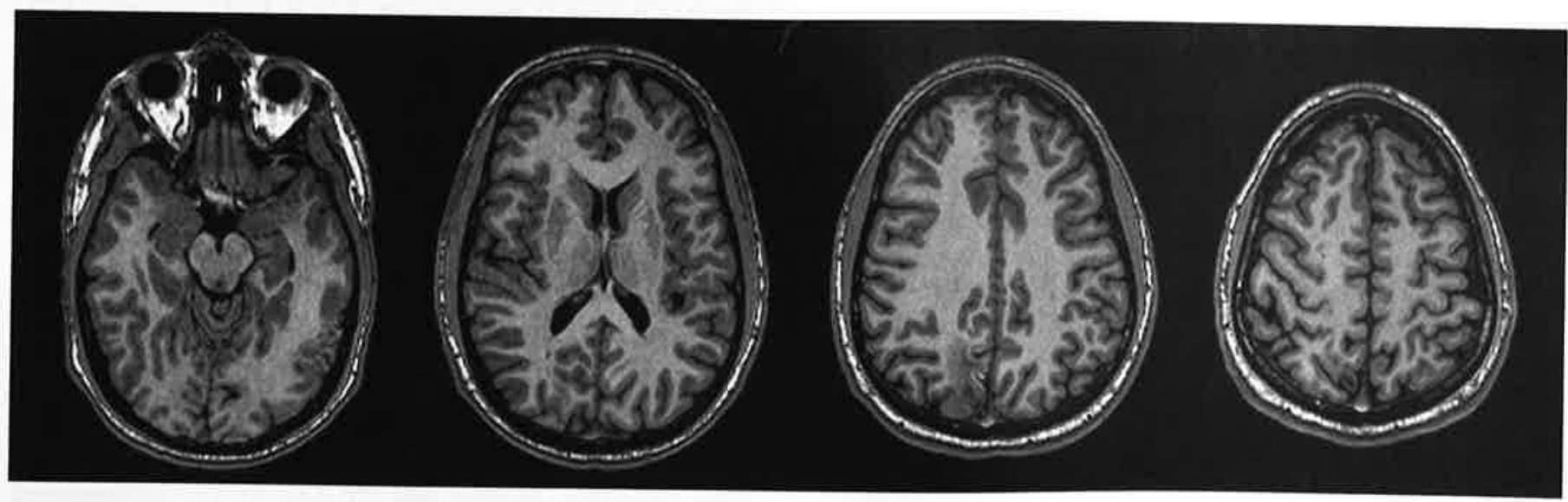
Figure 5: Seven-week follow up axial T2-weighted magnetic resonance images of the brain showing near-complete resolution of the previously seen
brain abscesses. 
microinfarctions in turn result in altered blood-brain barrier activity and subsequently serve as a nidus for infection. ${ }^{8}$ Direct support for altered blood-brain barrier function amongst patients with influenza is taken from rare human pathological studies, which identified significant vascular injury (vascular occlusion, microthrombi, and peri-vascular hemorrhage and edema) in the brains of patients with influenza encephalopathy.,10

Whether this blood-brain barrier injury results directly from viral infection and replication within endothelia or is secondary to an influenza-associated systemic inflammatory response is unclear. $^{11,12}$ We support the second, indirect mechanism of blood-brain barrier injury as few studies have found influenza virus genetic material within the brains of infected patients that died following associated central nervous system disease (e.g., influenza-virus associated encephalopathy). ${ }^{11}$ Animal studies suggest that influenza viruses gain entry to the central nervous system via the olfactory nerve and induce a potent neuroinflammatory response with liberation of multiple proinflammatory cytokines, including interleukin-1 beta (IL-1 beta), IL-6, IL-12, IL-18, and and TNF-alpha, which are prominent mediators of the acute phase response. ${ }^{13,14}$ This and other neuroinflammatory responses involving similar pro-inflammatory mediators are linked with potent alterations in blood-brain barrier permeability and biochemical function in several animal and human investigations. ${ }^{15}$ Such alterations in blood-brain barrier function dramatically reduce the protective function of the blood-brain barrier and we believe may have predisposed our patient to the development of brain abscesses.

Modern treatment for brain abscesses combines both medical and surgical management and includes antibiotics, stereotactic abscess aspiration, and total abscess excision via craniotomy. ${ }^{6,8}$ Antibiotics are either given empirically for six- to eight-weeks (often two-weeks of intravenous antibiotics followed by four- to six-weeks of oral) or are targeted against bacteria grown in blood cultures or abscess material obtained surgically. ${ }^{6.16}$ Medical management alone is considered when lesions are multiple, less than $3-\mathrm{cm}$ in diameter, located in eloquent brain areas, or if there are concomitant infections such as meningitis or ependymitis, especially when the target organism has been cultured. ${ }^{6}$ Surgical aspiration is often employed when there are multiple or deepseated lesions greater than $3-\mathrm{cm}$ in diameter, especially if periventricular in location. Craniotomy with complete abscess excision is a more morbid procedure only utilized in select situations, such as when there is increased intracranial pressure, traumatic brain injury, introduced foreign material, or if the lesion is located in the posterior fossa, among others. 6,8

The mortality rate from cerebral abscesses in the post-CT imaging era ranges from $17 \%$ to $32 \%$ with poorer outcomes being associated with lower GCS scores, immunosuppresion, and intraventricular rupture. ${ }^{6,17}$ Intraventricular rupture of a cerebral abscess is a devastating complication linked with a mortality rate of up to $84.5 \%$ in one series. ${ }^{6}$ Survivors of brain abscesses commonly suffer permanent neurological deficits, including hemiparesis and cognitive dysfunction. Seizures are a potential complication of brain abscesses that may present up to five-years later. Therefore, anti-seizure therapy is recommended for at least one-year following a brain abscess. Rates of recurrence range from $10 \%$ to $50 \%$ and thus serial imaging and close clinical follow-up is required. ${ }^{6}$ Of note, our patient had an excellent overall outcome and at 12 -weeks follow-up had no identifiable neurological deficits, was seizure-free, and was able to return to work.

In conclusion, we report the first case of a MSSA co-infection with the novel influenza A (H1N1) virus leading to multiple cerebral abscesses in a critically-ill patient treated successfully with medical therapy.

\section{REFERENCES}

1. Writing Committee of the WHO Consultation on Clinical Aspects of Pandemic (H1N1) 2009 Influenza. Clinical aspects of pandemic 2009 influenza A (H1N1) virus infection. N Engl J Med. 2010;362(18):1708-19.

2. Morens DM, Taubenberger JK, Fauci AS. Predominant role of bacterial pneumonia as a cause of death in pandemic influenza: implications for pandemic influenza preparedness. J Infect Dis. 2008;198(7):962-70.

3. Finelli L, Fiore A, Dhara R, et al. Influenza-associated pediatric mortality in the United States: increase of Staphylococcus aureus co-infection. Pediatrics. 2008;122(4):805-11.

4. Kumar A, Zarychanski R, Pinto R, et al. Critically ill patients with 2009 influenza A(H1N1) infection in Canada. JAMA. 2009;302 (17):1872-9.

5. Centers for Disease Control and Prevention. Bacterial co-infections in lung tissue specimens from fatal cases of 2009 pandemic influenza A (HIN1) -... United States, May-August, 2009. MMWR Morb Mortal Wkly Rep. 2009;58:1071-4.

6. Erdoğan E, Cansever T. Pyogenic brain abscess. Neurosurg Focus. 2008;24(6):E2.

7. Britt R, Enzmann D. Clinical stages of human brain abscess on serial CT scans after contrast infusion: computed tomographic, neuropathological, and clinical correlations. J Neurosurg. 1983; 59(6):972-89.

8. Schmidek HH. Suppurative intracranial infections. In: Winn HR, Youmans JR, editors. Youmans principles of neurological surgery. 5th ed. Philadelphia: WB Saunders; 2004. p. 1591-9.

9. Takahashi M, Yamada T, Nakashita Y, et al. Influenza virus-induced encephalopathy: clinicopathologic study of an autopsied case. Pediatr Int. 2000;42(2):204-14.

10. Sugaya N. Influenza-associated encephalopathy in Japan. Semin Pediatr Infect Dis. 2002;13(2):79-84.

11. Martin A, Reade EP. Acute necrotizing encephalopathy progressing to brain death in a pediatric patient with novel influenza $A$ (H1N1) infection. Clin Infect Dis. 2010;50(8);e50-2.

12. Vilensky JA, Foley $P$, Gilman S. Children and encephalitis lethargica: a historical review. Pediatr Neurol. 2007;37(2):79-84.

13. Leyva-Grado VH, Churchill L, Harding J, Krueger JM. The olfactory nerve has a role in the body temperature and brain cytokine responses to influenza virus. Brain Behav Immun. 2010;24(2):281-8.

14. Jang H, Boltz D, Sturm-Ramirez K, et al. Highly pathogenic H5NI influenza virus can enter the central nervous system and induce neuroinflammation and neurodegeneration. Proc Natl Acad Sci USA. 2009;106(33):14063-8.

15. Roberts DJ, Goralski KB. A critical overview of the influence of inflammation and infection on P-glycoprotein expression and activity in the brain. Expert Opin Drug Metab Toxicol. 2008;4 (10):1245-64.

16. Moorthy RK, Rajshekhar V. Management of brain abscess: an overview. Neurosurg Focus. 2008;24(6):E3.

17. Xiao F, Tseng MY, Teng L, Tseng HM, Tsai JC. Brain abscess: clinical experience and analysis of prognostic factors. Surg Neurol. 2005;63(5):442-50. 